\title{
Losartan ameliorates "upstream" pulmonary vein vasculopathy in a piglet model of pulmonary vein stenosis
}

\author{
Jiaquan Zhu, MD, PhD, ${ }^{\mathrm{a}, \mathrm{b}}$ Haruki Ide, MD, ${ }^{\mathrm{a}, \mathrm{b}}$ Yaqin Yana Fu, MD, ${ }^{\mathrm{a}, \mathrm{b}}$ Anouk-Martine Teichert, PhD, ${ }^{\mathrm{a}, \mathrm{b}}$ \\ Hideyuki Kato, MD, ${ }^{\mathrm{a}}$ Richard D. Weisel, MD, ${ }^{\mathrm{g}, \mathrm{h}}$ Jason T. Maynes, MD, PhD, ,d,e,f \\ John G. Coles, MD, ${ }^{\mathrm{a}, \mathrm{b}}$ and Christopher A. Caldarone, $\mathrm{MD}^{\mathrm{a}, \mathrm{b}}$
}

\begin{abstract}
Objectives: Pulmonary vein stenosis (PVS) is a relentless disease with a poor prognosis. Although surgical repair can effectively treat "downstream" (near left atrial junction) PVS, residual "upstream" (deep in lung parenchyma) PVS commonly dictates long-term survival. Our initial studies revealed an association between PVS and transforming growth factor- $\beta$ signaling, which led us to investigate the effect of losartan on upstream pulmonary vein vasculopathy in a piglet model of PVS.
\end{abstract}

\begin{abstract}
Methods: Neonatal Yorkshire piglets underwent sham surgical banding (sham, $n=6$ ), staged bilateral pulmonary vein banding of all pulmonary veins except the right middle pulmonary vein (banded, $n=6$ ), and staged pulmonary vein banding with losartan treatment (losartan, $1 \mathrm{mg} / \mathrm{kg} / \mathrm{d}, \mathrm{n}=7$ ). After 7 weeks, the hemodynamic data were obtained and the piglets killed.
\end{abstract}

Results: Pulmonary vein banding (compared with sham) was associated with continuous turbulent flow in banded pulmonary veins, pulmonary hypertension (pulmonary artery/systemic blood pressure ratio $0.51 \pm$ 0.06 vs $0.23 \pm 0.02, P<.001$ ), and diffuse pulmonary vein intimal hyperplasia in the upstream pulmonary veins $(P<.001)$. Losartan administration decreased the pulmonary artery/systemic blood pressure ratios compared with those in the banded piglets $(0.36 \pm 0.08$ vs $0.51 \pm 0.06, P=.007)$ but it remained greater than those in the sham group $(P=.001)$. Losartan was also associated with diminished pulmonary vein intimal hyperplasia compared with that in the banded piglets $(P<.001)$ but still remained more than that in the sham group $(P=.035)$. Pulmonary vein banding reduced vascular endothelial-cadherin expression, indicative of diminished endothelial integrity, which was restored with losartan.

Conclusions: Losartan treatment improved PVS-associated pulmonary hypertension and intimal hyperplasia and might be a beneficial prophylactic therapy for patients at high risk of developing PVS after pulmonary vein surgery. (J Thorac Cardiovasc Surg 2014;148:2550-8)

See related commentary on page 2559 .

Pulmonary vein stenosis (PVS) occurs in $\leq 18 \%$ of patients after repair of total anomalous pulmonary vein drainage, despite improved surgical techniques and perioperative

From the Division of Cardiovascular Surgery, ${ }^{\mathrm{a}}$ Labatt Family Heart Centre, and Departments of Anesthesia and Pain Medicine ${ }^{c}$ and Molecular Structure and Function, ${ }^{\mathrm{d}}$ The Hospital for Sick Children, Toronto, Ontario, Canada; Departments of Surgery, ${ }^{\mathrm{b}}$ Anesthesia, ${ }^{\mathrm{e}}$ and Biochemistry, ${ }_{\mathrm{f}}^{\mathrm{f}}$ University of Toronto, Toronto, Ontario, Canada; and Division of Cardiovascular Surgery ${ }^{\mathrm{g}}$ and Toronto General Research Institute, ${ }^{\mathrm{h}}$ Toronto General Hospital, University Health Network, Toronto, Ontario, Canada.

This study was supported by Canadian Institutes of Health Research (CIHR \# 312549) and the Saving Tiny Hearts Society.

Disclosures: Authors have nothing to disclose with regard to commercial support.

Read at the 94th Annual Meeting of The American Association for Thoracic Surgery, Toronto, Ontario Canada, April 26-30, 2014.

Received for publication April 9, 2014; revisions received June 26, 2014; accepted for publication July 16, 2014; available ahead of print Aug 27, 2014.

Address for reprints: Christopher A. Caldarone, MD, Division of Cardiovascular Surgery, Labatt Family Heart Centre, The Hospital for Sick Children, 555 University Ave, Toronto, ON M5G 1X8, Canada (E-mail: christopher.caldarone@ sickkids.ca)

$0022-5223 / \$ 36.00$

Copyright (c) 2014 by The American Association for Thoracic Surgery

http://dx.doi.org/10.1016/j.jtcvs.2014.07.050 management. ${ }^{1-4}$ PVS can also occur congenitally, with or without associated cardiac defects, and occasionally after nonpulmonary vein surgery. ${ }^{2,5-8}$ Surgical repair of PVS is often feasible when the stenosis is limited to the pulmonary vein-left atrial junction, but it has been associated with an increased risk of adverse composite outcomes (death, reoperation, or recurrent PVS), which can be as great as $30 \%$ to $45 \%{ }^{1,2,4}$ The current surgical techniques to repair PVS are limited, because they cannot directly address the upstream (deep in lung parenchyma) spread of pulmonary venous disease, an important mediator of long-term survival. With diffuse disease (eg, severe involvement of all 4 pulmonary veins), the 1-year mortality rate is $80 \%{ }^{2,3}$ As a last resort, palliative stenting of the pulmonary veins can be attempted, with a 1-year survival rate of only $62 \%$ and an incidence of in-stent stenosis of $63 \% .{ }^{9}$ Chemotherapy protocols have been suggested to treat diffuse PVS but have had limited success. ${ }^{10}$ Thus, currently, no therapy for upstream PVS is available.

We previously reported a piglet model of PVS associated with progressive diffuse obstructive intimal hyperplasia in the upstream pulmonary veins that recapitulates the clinical PVS pathogenesis. ${ }^{11}$ Specimens upstream from a banded 


\section{Abbreviations and Acronyms \\ AT1 receptor $=$ angiotensin II receptor, type 1 \\ PAP $=$ pulmonary artery pressure \\ PVS $\quad=$ pulmonary vein stenosis \\ $\mathrm{RV}=$ right ventricular \\ SMA $\quad=$ smooth muscle actin \\ TGF $-\beta=$ transforming growth factor $-\beta$ \\ VE-cadherin $=$ vascular endothelial cadherin \\ $\mathrm{vWF} \quad=$ von Willebrand factor}

pulmonary vein in the piglet model and human specimens from patients with PVS were associated with robust expression of transforming growth factor- $\beta$ (TGF- $\beta$ ). ${ }^{11}$

Losartan selectively blocks angiotensin II receptor, type 1 (AT1 receptor) and inhibits multiple avenues of TGF- $\beta$ action both directly and indirectly. ${ }^{12}$ Losartan inhibits TGF$\beta$-mediated activation of extracellular signal-regulated kinase $^{12}$ and Smad2 phosphorylation, both critical to the canonical signaling pathway of TGF- $\beta$. Losartan also decreases tissue expression of TGF- $\beta$ responsive genes and protein levels of connective tissue growth factor and collagen IV. ${ }^{12,13}$ Losartan restores the aortic architecture and root dimensions in a fibrillin-1-deficient mouse model of Marfan syndrome ${ }^{12,14}$ and is clinically available for the prevention of dilated aortopathy associated with Marfan syndrome. ${ }^{13,15}$ The relationship between losartan and pulmonary vein pathology, however, has not been reported. We hypothesized that losartan might slow the progression of upstream disease in our piglet model of PVS.

\section{METHODS}

The Animal Care Committee at the Hospital for Sick Children approved the present study. Neonatal piglets $(3.7 \pm 0.6 \mathrm{~kg})$ were divided into 3 groups: sham surgical banding (sham, $\mathrm{n}=6$ ), pulmonary vein banding (banded, $\mathrm{n}=6$ ), and banded with losartan treatment (losartan, $\mathrm{n}=7$ ).

\section{PVS Model and Hemodynamic Measurement}

The pulmonary vein banding procedure was modified from that of our previous study. ${ }^{11}$ Each piglet underwent a 2 -stage banding procedure involving bilateral thoracotomies. In the stage I procedure, the left upper pulmonary vein and the lower common pulmonary vein were banded through the left fifth intercostal space. After a 1-week recovery period, the stage II procedure included banding of the right upper pulmonary vein through the fourth intercostal space, and the right middle pulmonary vein was left unbanded. The unbanded right middle pulmonary vein (which drains a relatively small portion of the right lung) provided an intra-animal control for the banded pulmonary veins and improved survivability in the model. Cotton umbilical tapes $(0.125$-in. width, and length equivalent to 1.3 times the pulmonary vein circumference) were used to band the pulmonary veins. The sham group underwent identical procedures, including encirclement of the pulmonary veins, with the exception that the bands were not left in place. In the losartan group, $1 \mathrm{mg} / \mathrm{kg} / \mathrm{d}$ losartan (Cozaar; Merck, Whitehouse Station, NJ) was administered orally from the second postoperative day after the stage I procedure to the day before tissue harvest.

At 7 weeks after the stage II procedure, the pigs underwent a hemodynamic assessment and tissue harvest. The body surface area was calculated using the following formula ${ }^{16}$ : body surface area $\left(\mathrm{m}^{2}\right)=0.0734 \times(\text { body weight })^{0.656}$. The heart rate, invasive arterial blood pressure, and saturation were monitored. Echocardiography was performed. A 5F Swan-Ganz catheter (Teleflex, Limerick, Pa) was inserted from the right internal jugular vein to measure the central venous pressure, right ventricular (RV) pressure, pulmonary artery pressure (PAP), and pulmonary capillary wedge pressure. Cardiac output was measured by thermodilution (model 9520A, Edwards Lifesciences, Irvine, Calif). After sternotomy, the RV pressure, left ventricular pressure, and left atrial pressure were obtained by direct needle measurement. The heart and lungs were removed en bloc quickly after exsanguination.

The pulmonary veins were dissected as distally as possible from the pulmonary vein-left atrial junction and divided into 3 equivalent segments if long enough. Otherwise, the pulmonary veins were divided into 2 segments. The proximal segment was closest to the site of pulmonary vein banding (1-3 cm from the site of banding) and was termed "downstream." The adjacent "upstream" segments originated $>3 \mathrm{~cm}$ from the site of banding and extended $\leq 8 \mathrm{~cm}$ into the lung. The heart was then harvested, and the ventricle was split into 2 parts: the RV free wall and the left ventricle.

\section{Histologic Examination}

Paraffin-embedded pulmonary vein tissues were sectioned and stained with hematoxylin and eosin and trichrome staining. Movat pentachrome staining was performed to quantify intimal hyperplasia in every segment. ImageJ (National Institutes of Health, Bethesda, Md) was used for the quantification. The intimal area was measured between the endothelial layer and lamina interna, and the media area was quantified from the lamina interna to the outside border of the smooth muscle cells. The circumference of the internal lamina was used to calculate the radius of this vessel.

The extent of intimal hyperplasia is reported using the ratio of the intima to media area. However, this ratio can lead to inaccurate interpretation in specimens with irregular hypertrophy contours. A complimentary method was used as a second index to quantify intimal hyperplasia that normalized the measured intimal area to the square of the calculated radius, representing the ratio of the intimal area to an idealized vessel internal luminal area. A third technique measured the media area and normalized this measurement to the square of the calculated radius (media area/ $\mathrm{R}^{2}$ ) to evaluate the extent of media hypertrophy. All measurements were performed by an observer who was unaware of the specimen grouping.

\section{Immunofluorescence}

Paraffin-embedded pulmonary vein slides were deparaffinized in xylene and rehydrated in ethanol. Antigens were re-exposed with sodium citrate buffer (Dako, Glostrup, Denmark), immersed in blocking buffer for $40 \mathrm{mi}$ nutes, incubated with primary antibodies for 2 hours, washed in phosphatebuffered saline solution, and labeled with secondary fluorophore-labeled antibodies. The nucleus was stained with $46^{\prime}$-diamidino-2-phenylindole$2 \mathrm{HCl}(1: 1000)$.

The endothelial markers evaluated included CD31 (1:100; Abcam, Cambridge, Mass), vascular endothelial cadherin (VE-cadherin, 1:50; Thermo Fisher Scientific, Waltham, Mass), and von Willebrand factor (vWF, 1:100; Dako Canada Inc, Burlington, Ontario, Canada). The mesenchymal markers evaluated included fibronectin (1:100; BD Transduction Laboratories, Franklin Lakes, NJ) and $\alpha$-smooth muscle actin ( $\alpha$-SMA, 1:50; Santa Cruz Biotechnology, Santa Cruz, Calif).

\section{Protein Extraction and Western Blotting}

Left upper pulmonary vein samples were homogenized in lysis buffer and centrifuged (20 minutes, $13,400 \mathrm{~g}, 4^{\circ} \mathrm{C}$ ), and supernatants were collected. The protein concentrations were quantified using a Bradford protein assay (Bio-Rad, Hercules, Calif). The samples were separated on midi-Protein TGX Precast Gels (Bio-Rad), transferred to nitrocellulose membranes, and blocked with $5 \%$ skim milk or $5 \%$ bovine serum albumin for 1 hour. The membranes were probed with antibodies of interest, and the blots were then incubated with goat anti-mouse IgG horseradish peroxidase 
TABLE 1. Hemodynamics and right ventricular hypertrophy

\begin{tabular}{|c|c|c|c|c|}
\hline \multirow[b]{2}{*}{ Variable } & \multicolumn{3}{|c|}{ Group } & \multirow[b]{2}{*}{$P$ value } \\
\hline & Sham $(n=6)$ & Banded $(n=6)$ & $\operatorname{Losartan}(n=7)$ & \\
\hline HR (beats/min) & $99.8 \pm 10.9$ & $117.2 \pm 16.9^{*}$ & $105.6 \pm 12.7$ & .113 \\
\hline Systolic PAP (mm Hg) & $16.2 \pm 2.1$ & $35.3 \pm 3.1^{*}$ & $26 \pm 7^{*}, \dagger$ & $<.001$ \\
\hline Diastolic PAP (mm Hg) & $9.3 \pm 1.9$ & $20 \pm 3.3^{*}$ & $14.9 \pm 4.1^{*}, \dagger$ & $<.001$ \\
\hline Mean PAP (mm Hg) & $12 \pm 2.4$ & $26.8 \pm 2.6^{*}$ & $19.9 \pm 7 *, \dagger$ & $<.001$ \\
\hline Systolic ABP & $70.5 \pm 7.9$ & $69.7 \pm 9.9$ & $72 \pm 5.5$ & .863 \\
\hline Mean ABP & $51.7 \pm 8.4$ & $55 \pm 7.2$ & $57 \pm 3.3$ & .394 \\
\hline Systolic PAP/ABP & $0.23 \pm 0.02$ & $0.51 \pm 0.06^{*}$ & $0.36 \pm 0.08^{*}, \dagger$ & $<.001$ \\
\hline Systolic RVP/LVP & $0.3 \pm 0.02$ & $0.57 \pm 0.1^{*}$ & $0.42 \pm 0.05^{*}, \dagger$ & $<.001$ \\
\hline CVP (mm Hg) & $1.8 \pm 1.5$ & $2.7 \pm 1$ & $4.1 \pm 1^{*}, \dagger$ & .01 \\
\hline PCWP (mm Hg) & $5.3 \pm 2.3$ & $13.9 \pm 2.7^{*}$ & $12.5 \pm 2.3^{*}$ & $<.001$ \\
\hline Mean LAP (mm Hg) & $5.3 \pm 2.3$ & $5.7 \pm 1.8$ & $6.5 \pm 1.8$ & .531 \\
\hline PVR index $\left(\right.$ Wood $\left.\times \mathrm{m}^{2}\right)$ & $0.98 \pm 0.19$ & $3.91 \pm 0.84^{*}$ & $2.58 \pm 1.48^{*}, \dagger$ & .001 \\
\hline SVR index $\left(\right.$ Wood $\left.\times \mathrm{m}^{2}\right)$ & $7.51 \pm 1.49$ & $9.51 \pm 1.38$ & $10.07 \pm 2.26 \dagger$ & .055 \\
\hline Mean CI $\left(\mathrm{L} / \mathrm{min} / \mathrm{m}^{2}\right)$ & $6.73 \pm 1.1$ & $5.62 \pm 1.2$ & $5.34 \pm 0.98^{*}$ & .8 \\
\hline $\mathrm{SV}$ index $\left(\mathrm{mL} / \mathrm{m}^{2}\right)$ & $67.98 \pm 12.25$ & $47.69 \pm 5.74^{*}$ & $51.78 \pm 13.96^{*}$ & .017 \\
\hline $\mathrm{RV}$ mass index $\left(\mathrm{g} / \mathrm{m}^{2}\right)$ & $50.23 \pm 4.16$ & $71.35 \pm 12.84^{*}$ & $54.22 \pm 11.87 \dagger$ & .007 \\
\hline LV mass index $\left(\mathrm{g} / \mathrm{m}^{2}\right)$ & $139.4 \pm 8$ & $129.9 \pm 7.2$ & $126.7 \pm 21$ & .286 \\
\hline $\mathrm{RV} / \mathrm{LV}$ weight & $0.36 \pm 0.02$ & $0.55 \pm 0.09^{*}$ & $0.43 \pm 0.09 \dagger$ & .002 \\
\hline LVSF $(\%)$ & $36.3 \pm 4.5$ & $34.5 \pm 5.1$ & $36.2 \pm 4.5$ & NS \\
\hline
\end{tabular}

Data presented as mean \pm standard deviation. $H R$, Heart rate; $P A P$, pulmonary artery pressure; $A B P$, arterial blood pressure; $R V P$, right ventricular pressure; $L V P$, left ventricular pressure; $C V P$, central venous pressure; $P C W P$, pulmonary capillary wedge pressure; $L A P$, left atrial pressure; $P V R$, pulmonary vascular resistance; $S V R$, systemic vascular resistance; $C I$, cardiac index; $S V$, stroke volume; $R V$, right ventricular; $L V$, left ventricular; $L V S F$, left ventricular shortening fraction; $N S$, not significant. $* P<.05$ versus sham. $\dagger P<.05$ versus banded.

(1:5000) and goat anti-rabbit IgG horseradish peroxidase (1:5000) secondary antibodies. The membranes were developed using Clarity Western ECL substrate (Bio-Rad). Densitometry was performed using Quantity One and Image Lab analysis software (Bio-Rad). Glyceraldehyde-3-phosphate dehydrogenase was used as a loading control and to normalize the data.

The primary antibodies included CD31 (1:500; Abcam), VE-cadherin (1:1000; Thermo Fisher Scientific), and vWF (1:500; Dako Canada Inc). The mesenchymal markers evaluated included fibronectin (1:1000; BD Transduction Laboratories) and $\alpha$-SMA (1:2000; Santa Cruz Biotechnology). TGF- $\beta$ (1:1000; Abcam) and phosphorylated SMAD2 (1:800, Cell Signaling, Denvers, Mass) were also quantified.

\section{Statistical Analysis}

Continuous data are presented as the mean \pm standard deviation. Oneway analysis of variance or a nonparametric comparison was used to compare the hemodynamic data, histologic scores, and semiquantification of Western blotting among the 3 groups. A post hoc comparison was analyzed using Fisher's least significant difference test or Dunnett's test, depending on the equality of the variance. The Mann-Whitney $U$ test and Kruskal-Wallis $\mathrm{H}$ test were used for nonparametric comparisons, as appropriate. The data were analyzed using SPSS, version 19 (IBM Corp, Armonk, NY).

\section{RESULTS}

\section{Losartan Treatment Decreased Banding-Induced Pulmonary Artery Hypertension and RV Hypertrophy}

The hemodynamic data are summarized in Table 1. Pulmonary vein banding induced an increase in the heart rate, PAP, pulmonary capillary wedge pressure, systolic PAP/ arterial blood pressure, systolic RV pressure/left ventricular pressure, and pulmonary vascular resistance index $(P<.05$ for all vs sham). The cardiac index and stroke volume index were lower than those in the sham group. Banding also induced RV hypertrophy, with a greater RV mass index and $\mathrm{RV} /$ left ventricular weight ratio $(P<.05$ for both vs sham).

Losartan treatment diminished the systolic PAP/arterial blood pressure and pulmonary vascular resistance index compared with the banded groups $(P<.001$ and $P=.045)$, but these parameters had not entirely normalized compared with the sham group $(P=.001$ and $P=.015)$. Losartan administration did not improve the pulmonary capillary wedge pressure, cardiac index, or stroke volume index compared with the banded group. Pulmonary vein banding produced robust RV hypertrophy (RV mass index) compared with the sham group $(P=.003)$. Losartan prevented the RV hypertrophy seen in the banded group $(P=.01$ for banded vs losartan) and reduced the RV hypertrophy to a level that was not significantly different statistically than that in the sham group $(P=.503$; losartan vs sham).

\section{Losartan Reduced Diffuse Intimal Hyperplasia in Banded Pulmonary Veins}

The histologic alterations associated with pulmonary vein banding and losartan treatment are shown in Figure 1. Hematoxylin and eosin staining (Figure 1, A-E) demonstrated a single cell layer of endothelial cells on the 
Upstream Banded PVs Banded
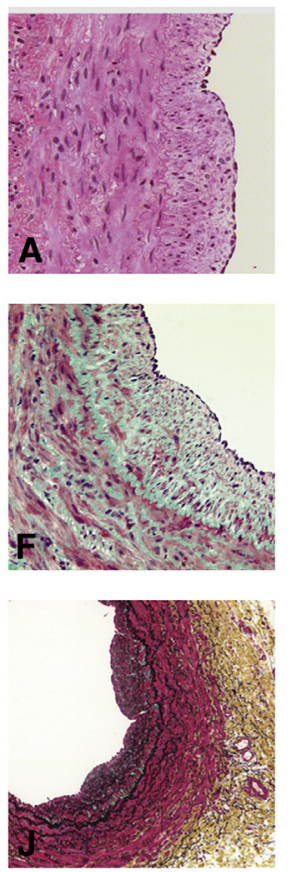

Losartan
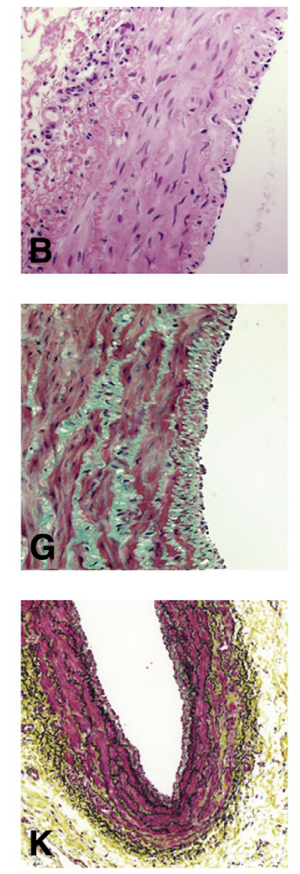

Unbanded RMPV
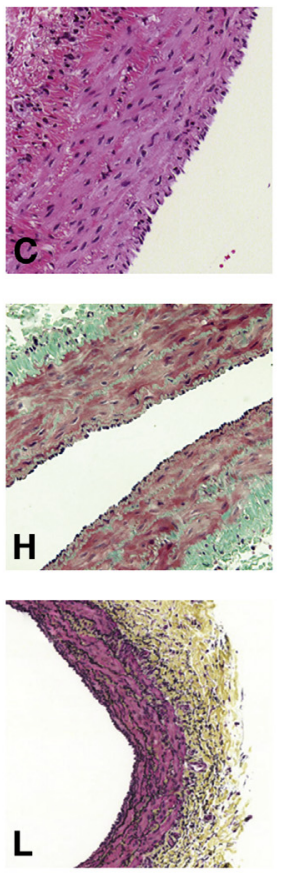

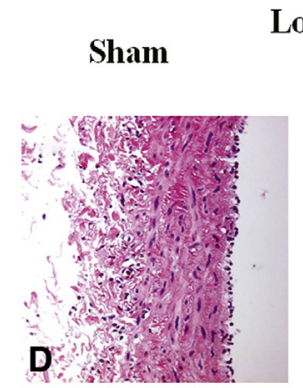

Longitudinal Section of Banded PV

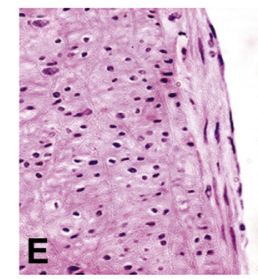

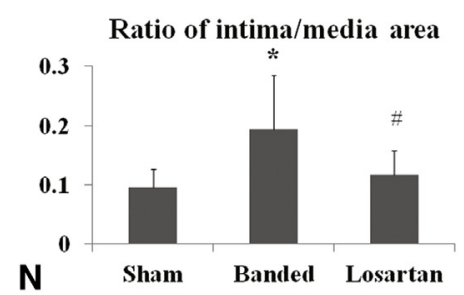
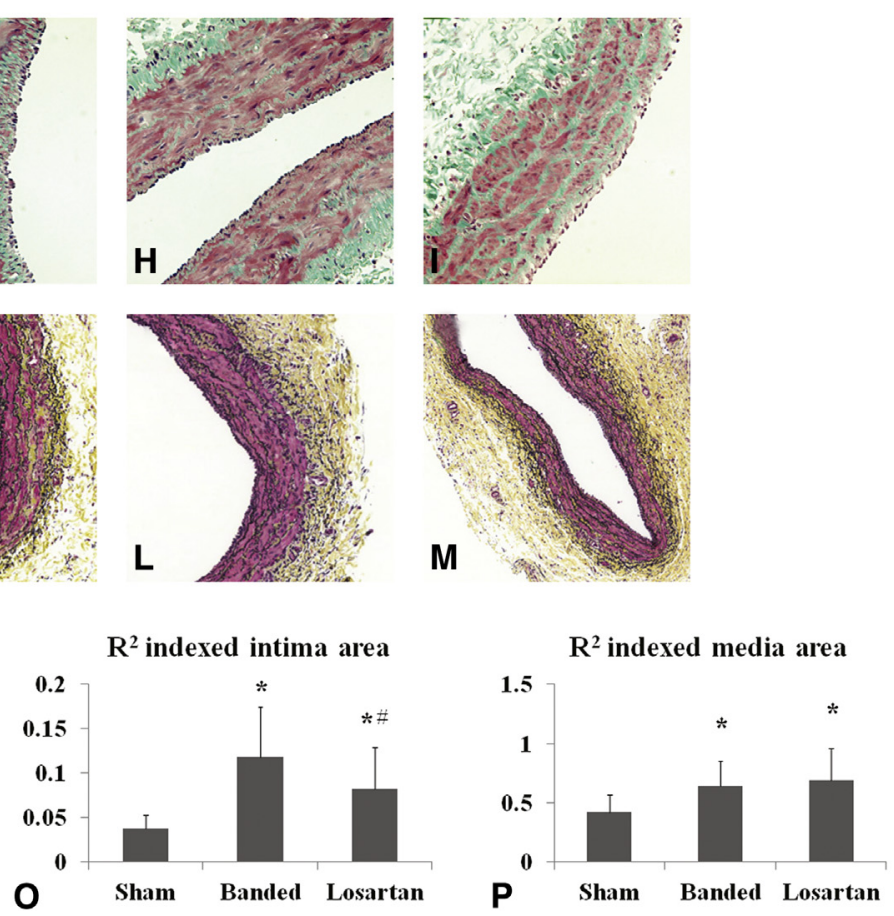

FIGURE 1. Histologic findings of upstream pulmonary veins $(P V s)$ after PV banding. A-E, Hematoxylin and eosin staining, F-I, trichrome staining, and J-M, Movat staining. A, F, J, The upstream banded PVs in the banded group had significantly greater intimal hyperplasia compared with D, I, M, the sham group. C, H, L, The unbanded right middle PV $(R M P V)$ appeared normal. B, G, K, Losartan remarkably ameliorated this pathologic change, N, O, quantified by both the ratio of intima/media area and the square of the radius indexed intima area. P, However, the square of the radius indexed media area was similar between the banded and losartan groups. R, Virtual radius calculated from the circumference of the internal elastic lamina. $* P<.05$ versus sham; $\# P<.05$ versus banded.

internal elastic lamina in the sham group. However, significant intimal hyperplasic cells were found in the upstream pulmonary veins in the banded and losartan groups both. The nuclei of the cells in the neointima were spindle-like in both the cross-sectional (Figure 1, $A-D$ ) and longitudinal hematoxylin and eosin (Figure 1,E) sections. Trichrome staining (Figure 1, F-I) indicated collagen deposition and excessive cell accumulation in the neointima. These findings were also confirmed by Movat pentachrome staining (Figure 1, $J-M$ ). Although these indexes were evident in the banded pulmonary vein, they were not seen in the unbanded right middle pulmonary vein.

The extent of the hyperplasia is summarized in Figure 1, $N-P$. The ratio of the intima/media in the upstream pulmonary veins of the banded group was significantly greater than that of the sham groups $(P<.001$; Figure $1, N)$. Losartan significantly protected the pulmonary vein from intimal hyperplasia $(P<.001)$, although not quite to the level found in the sham group $(P=.09$; Figure $1, N)$. The $\mathrm{R}^{2}$ indexed intimal area was also significantly increased in the banded compared with the control groups $(P<.001$; Figure $1, O)$. The losartan group had a smaller $\mathrm{R}^{2}$ indexed intima area than did the banded group $(P=.035)$, but it was still larger than that of the sham group $(P<.001)$. The $\mathrm{R}^{2}$ indexed media area (Figure $1, P$ ) was significantly greater in the banded $(P<.001)$ and losartan $(P<.001)$ groups compared with the sham group. However, no difference was found between the banded and losartan groups $(P=.384)$. No differences in intimal hyperplasia were found among the different banded branches or segments among the groups $(P>.05$ for all). 


\section{Losartan Treatment Maintained VE-Cadherin Expression in Upstream Banded Pulmonary Veins}

Representative immunofluorescence images of the banded upstream pulmonary veins are shown in Figure 2, $A-I$. Endothelial markers, including CD31, vWF, and VEcadherin, were expressed in the endothelial layer but not in the subendothelial area of the neointima, which was $\alpha$ SMA positive.

Quantification of protein expression in the pulmonary veins by Western blotting indicated significantly lower levels of VE-cadherin and CD31 expression in the banded group compared with the sham group $(P=.045$ and $P=.021$, respectively; Figure $2, J$ and $K$ ). Losartan treatment prevented the decrease of VE-cadherin expression compared with the banded group $(P=.004$; Figure $2, J)$. Losartan did not prevent the loss of CD31 expression after banding $(P=.647$; Figure $2, K)$. No differences were found in the protein expression of TGF- $\beta$, phosphorylated SMAD2, vWF, $\alpha$-SMA, and fibronectin (all $P>.05$ within groups, all comparisons).

\section{DISCUSSION}

Our pulmonary vein banded piglet model recapitulates important clinical manifestations of PVS with diffuse upstream intimal hyperplasia in the banded pulmonary veins, pulmonary hypertension, and right ventricular hypertrophy. Postoperative losartan treatment significantly decreased PVS-associated pulmonary hypertension and pulmonary vein intimal hyperplasia. Protein expression of VEcadherin was decreased after pulmonary vein banding; and losartan treatment maintained VE-cadherin levels close to the levels found in the sham group.

\section{Pathologic Changes in Upstream Banded Pulmonary Vein: Clinical Relevance}

Intimal hyperplasia was evident in the upstream banded pulmonary vein, as described previously by $\mathrm{us}^{11}$ and others. ${ }^{17}$ We found an accumulation of $\alpha$-SMA+/CD31cells and collagen deposition in the neointima between the endothelial layer and internal elastic lamina. A previous report also demonstrated increased SMA+ cell accumulation and collagen deposition in the subendothelial lesions of banded pulmonary veins. ${ }^{18}$ These pathologic changes are consistent with those seen in patients with PVS. ${ }^{19-22}$ Sadr and colleagues ${ }^{21}$ and Riedlinger and colleagures ${ }^{22}$ found abnormal intimal spindle-shaped cell proliferation within a loose acid mucopolysaccharide matrix in the stenotic pulmonary veins near the left atrial junction in infants with primary PVS. These abnormal cells were positive for SMA, muscle-specific actin, vimentin, and collagen but were negative for CD31 and CD34, suggesting that myofibroblasts are the culprit cells in PVS. Rough endoplasmic reticulum, indented nuclei, pinocytotic vesicles, and cytoplasmic microfilaments were identified by electron microscopy, further supporting their identity as myofibroblasts. Intimal fibrosis, medial thickening, and ectasia of the lymphatics has also been shown within the lungs of patients with congenital mitral stenosis and cor triatriatum by Endo and colleagues. ${ }^{20}$ Together, these findings indicate that intimal hyperplasia with myofibroblast accumulation is the main histopathologic manifestation in the upstream stenotic pulmonary veins. Our piglet model recapitulated these clinical pathologic findings from PVS and was used to investigate potential treatments, such as losartan.

\section{Potential Mechanisms for Beneficial Effects of Losartan in PVS}

The expression of the endothelial markers, VE-cadherin and CD31, decreased in the banded pulmonary veins, suggesting an important role for endothelial cell dysfunction in the development of PVS. Moreover, a third endothelial cell-specific marker, vWF, remained unchanged. The dramatic decrease in VE-cadherin levels in the banded group was prevented by losartan treatment, and these levels were maintained at nearly normal levels. This effect of losartan would seem to be very specific to VE-cadherin and not a generalized influence on endothelial cells per se, given the findings for CD31 and vWF (ie, other endothelial cellspecific markers were not affected).

The endothelium is a barrier between the intravascular and extravascular spaces, and its integrity is essential to maintain normal vascular health. After banding, pulmonary vein flow becomes turbulent, and increasing shear stress could perturb the endothelium. Later, the pulmonary veins stretch in response to the increasing pressures. Mechanical forces on the endothelial cells could contribute to alterations in endothelial cell integrity and activation of signaling pathways. ${ }^{23-25}$ In the present study, losartan reduced the neointimal hyperplasia and the resultant pulmonary hypertension, and we speculate that the losartan might have acted through preservation of the pulmonary vein endothelial integrity.

Losartan protected pulmonary vein endothelium from banding-induced decrements in VE-cadherin expression. VE-cadherin is a component of endothelial cell-to-cell adherens junctions with an essential role in the control of vascular permeability. ${ }^{24}$ Decreased VE-cadherin levels have been associated with dissociation of cell junctions, which facilitates the entry of circulating leukocytes and progenitor cells into the subendothelial space, promoting inflammatory responses and cell proliferation. ${ }^{24}$ Importantly, VE-cadherin interacts with multiple growth factor receptors, such as the TGF- $\beta$ receptor, and modulates their downstream signaling, including interactions with pericytes and smooth muscle cells and cell polarity. ${ }^{23,24,26}$ In our study, the preservation of VE-cadherin levels after banding with losartan treatment was associated with diminished 


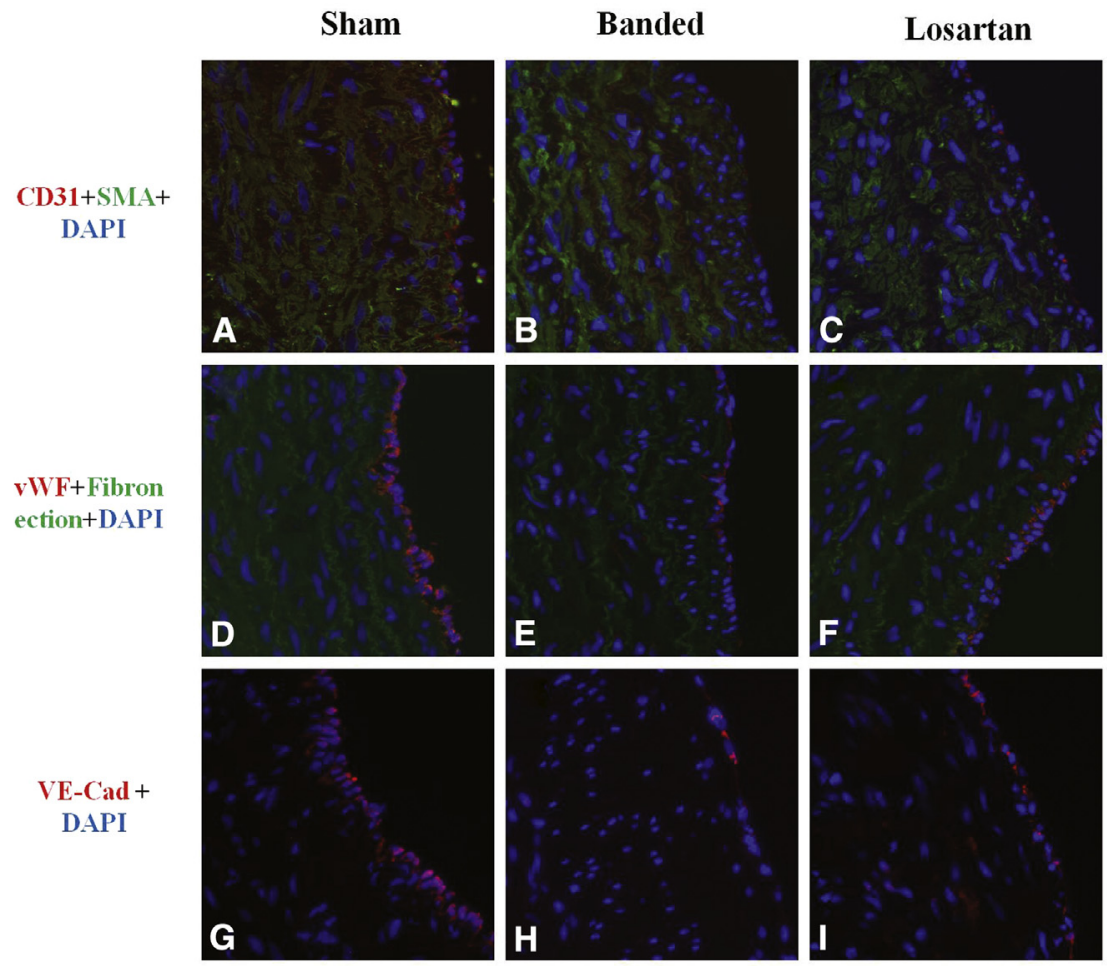

VE-Cad/GAPDH

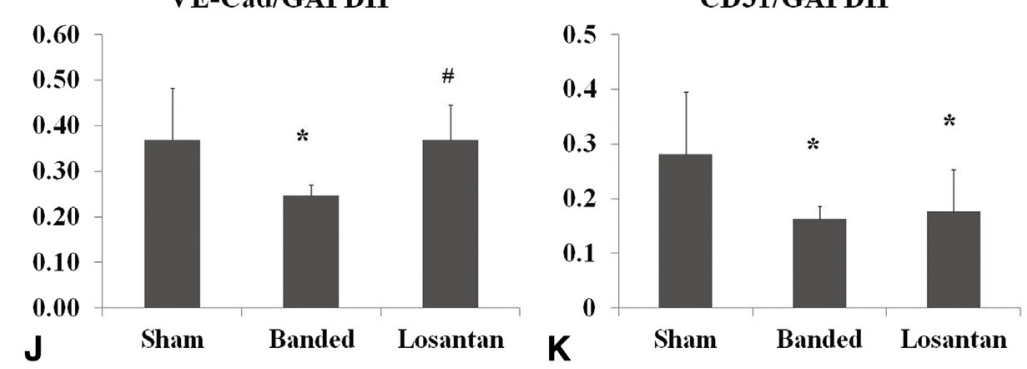

FIGURE 2. Immunofluorescence staining and Western blots of the upstream pulmonary veins. Immunofluorescence staining for $\mathrm{A}$ to $\mathrm{C}$, $\mathrm{CD} 31+\alpha$-smooth muscle actin $(S M A)$, D-F, von Willebrand factor $(v W F)+$ fibronection, and G to I, vascular endothelial-cadherin (VE-Cad) in upstream pulmonary veins. J, K, VE-cadherin and CD31 levels were both significantly decreased in the banded group, and losartan maintained the VE-cadherin levels, shown by Western blotting. DAPI, 46'-Diamidino-2-phenylindole-2 $\mathrm{HCl}$; $G A P D H$, glyceraldehyde-3-phosphate dehydrogenase. $* P<.05$ versus sham; \#P<.05 versus banded.

intimal hyperplasia. From the present findings, we speculate that losartan maintains the integrity of the endothelium through a positive interaction with VE-cadherin, thereby slowing the progression of PVS. Losartan has other direct and indirect effects that could also influence PVS progression. However, the precise downstream signaling pathways responsible for the preservation of VE-cadherin and the protective effects of losartan require additional investigation. In Figure 3, we propose a general model depicting the interaction of losartan in the pathogenesis of PVS.

\section{TGF- $\beta$ Signaling in PVS}

We did not detect differences in the TGF- $\beta$ levels among the groups in the present study, in contrast to our previous study, which demonstrated greater TGF- $\beta$ levels in the banded pulmonary veins (and provided the rationale to test losartan). Furthermore, we found unchanged protein levels for phosphorylated SMAD2, a key downstream mediator in canonical TGF- $\beta$ signaling. This puzzling discrepancy could be related to differences in the models. First, in the present model, the right middle pulmonary vein was left unbanded, which might have resulted in less severe pulmonary venous obstruction and diminished stimulation of TGF- $\beta$ expression. The mean pulmonary artery pressures in the present study tended to be slightly lower than those in our previous study $(26.8 \pm 2.6$ vs $34.3 \pm 8.9 \mathrm{~mm} \mathrm{Hg}$, $P=.076$ ), suggesting a potential difference in the severity of pulmonary venous obstruction. Second, the upstream 


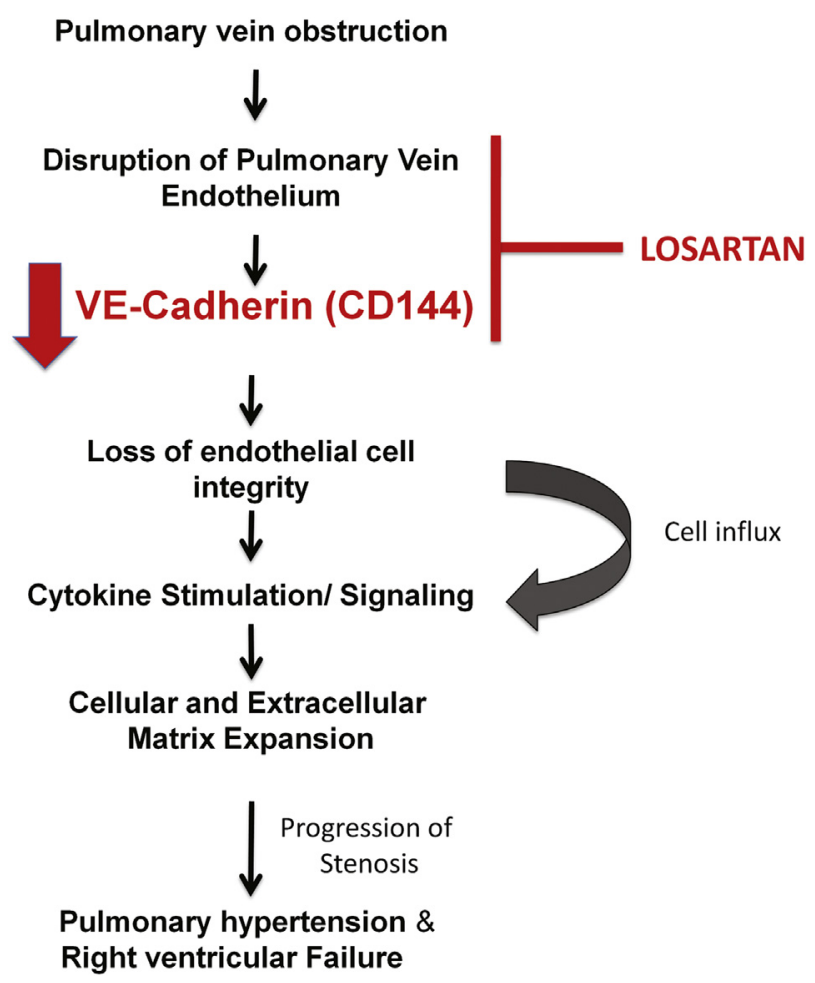

FIGURE 3. Proposed model of pulmonary vein stenosis pathogenesis and losartan effect. $V E$, Vascular endothelial.

banded pulmonary veins harvested in the present study were more distal ("upstream") than the vein segments evaluated in the previous study. Possibly, the extent of TGF- $\beta$ expression is diminished when sampled further from the site of pulmonary vein banding. Finally, it is possible that the elevated TGF- $\beta$ levels occurred as an early signaling event after banding that we failed to capture at the 7-week measurement point used in the present study.

Although we did not find a difference in the expression of TGF- $\beta$ in the present study, we can speculate on alternative mechanisms whereby losartan might be efficacious through pathways independent of TGF- $\beta$. For example, the work of others has suggested that autoantibodies against AT1 receptors might affect vascular endothelial integrity in preeclampsia, which is blocked by losartan. ${ }^{27}$ In the present study, losartan-dependent preservation of vascular integrity was suggested by the increased VE-cadherin levels in the losartan-treated piglets. Although beyond the scope of the present study, the close proximity of the AT1 receptors to the extracellular domains of VE-cadherin allows us to speculate that a potential "stabilizing" association exists between VE-cadherin and losartan-mediated interaction with AT1 receptors. To better study the relationship among losartan, AT1 receptors, and VE-cadherin expression, we are developing a mouse model of pulmonary vein stenosis that will enable us to use appropriate transgenic models and elucidate the mechanisms of losartan efficacy.

\section{Losartan Dosage}

We administered a losartan dosage of $1 \mathrm{mg} / \mathrm{kg} / \mathrm{d}$, which is within the recommended range for clinical usage and similar to the dose used in studies of Marfan syndrome. $^{28-30}$ No hypotension or other significant side effects were observed, indicating a positive safety profile for this drug in the setting of PVS. Nevertheless, an appropriately powered dose-response study will be important to determine the optimal dosage in terms of preventing progression of PVS.

\section{PVS, Losartan, and Right-Sided Heart Failure}

A prominent feature of end-stage PVS is right-sided heart failure. Friedberg and colleagues ${ }^{31}$ recently found losartan abrogated adverse ventricular remodeling in isolated right ventricular hypertension by inhibiting the TGF- $\beta$ pathway. Therefore, it is possible that losartan could potentially have synergistic beneficial effects in patients with end-stage PVS through amelioration of PVS and associated right-sided heart failure.

\section{Study Limitations}

A major limitation in the interpretation of our study lies in the potential for translation into clinical practice. In our model, losartan was administered before the development of pulmonary venous hypertension (the bands were not constrictive at implantation). Thus, our model explored the effect of losartan as a prophylactic therapy before the establishment of upstream PVS. The present data would support the use of losartan at pulmonary vein surgery in nonobstructed pulmonary veins (eg, straightforward repair of total anomalous pulmonary venous drainage). Our data do not, however, allow direct extrapolation to the more concerning clinical scenario in which a surgeon operates to relieve established PVS. ${ }^{3}$ We will, therefore, initiate additional studies in a piglet model of chronic PVS to test the hypothesis that losartan can be used to treat established upstream PVS.

\section{CONCLUSIONS}

Pulmonary hypertension and intimal hyperplasia extending upstream into the lung were found in our PVS piglet model. Losartan treatment ameliorated progression of PVS-associated pulmonary hypertension and intimal hyperplasia, possibly through VE-cadherin-associated maintenance of endothelial integrity. Losartan could be useful as prophylactic therapy after surgical repair of pulmonary vein anomalies.

We greatly thank Cameron Slorach and Wei Hui for the echocardiography examinations and Marvin Estrada and Anson Lam for the animal preparations. 


\section{References}

1. Ricci M, Elliott M, Cohen GA, Catalan G, Stark J, de Leval MR, et al. Management of pulmonary venous obstruction after correction of TAPVC: risk factors for adverse outcome. Eur J Cardiothorac Surg. 2003;24:28-36.

2. Hickey EJ, Caldarone CA. Surgical management of post-repair pulmonary vein stenosis. Semin Thorac Cardiovasc Surg Pediatr Card Surg Annu. 2011;14: $101-8$.

3. Yun TJ, Coles JG, Konstantinov IE, Al-Radi OO, Wald RM, Guerra V, et al. Conventional and sutureless techniques for management of the pulmonary veins: evolution of indications from postrepair pulmonary vein stenosis to primary pulmonary vein anomalies. J Thorac Cardiovasc Surg. 2005;129:167-74.

4. Devaney EJ, Ohye RG, Bove EL. Pulmonary vein stenosis following repair of total anomalous pulmonary venous connection. Semin Thorac Cardiovasc Surg Pediatr Card Surg Annu. 2006;9:51-5.

5. Breinholt JP, Hawkins JA, Minich LA, Tani LY, Orsmond GS, Ritter S, et al. Pulmonary vein stenosis with normal connection: associated cardiac abnormalities and variable outcome. Ann Thorac Surg. 1999;68:164-8.

6. Devaney EJ, Chang AC, Ohye RG, Bove EL. Management of congenital and acquired pulmonary vein stenosis. Ann Thorac Surg. 2006;81:992-5; discussion 995-6.

7. Latson LA, Prieto LR. Congenital and acquired pulmonary vein stenosis. Circulation. 2007:115:103-8.

8. Viola N, Alghamdi AA, Perrin DG, Wilson GJ, Coles JG, Caldarone CA. Primary pulmonary vein stenosis: the impact of sutureless repair on survival. J Thorac Cardiovasc Surg. 2011;142:344-50.

9. Balasubramanian S, Marshall AC, Gauvreau K, Peng LF, Nugent AW, Lock JE, et al. Outcomes after stent implantation for the treatment of congenital and postoperative pulmonary vein stenosis in children. Circ Cardiovasc Interv. 2012;5: 109-17.

10. Rehman M, Jenkins KJ, Juraszek AL, Connor JA, Gauvreau K, Muneeb M, et al. A prospective phase II trial of vinblastine and methotrexate in multivessel intraluminal pulmonary vein stenosis in infants and children. Congenit Heart Dis. 2011;6:608-23.

11. Kato H, Fu YY, Zhu J, Wang L, Aafaqi S, Rahkonen O, et al. Pulmonary vein stenosis and the pathophysiology of "upstream" pulmonary veins. J Thorac Cardiovasc Surg. 2014;148:245-53.

12. Habashi JP, Doyle JJ, Holm TM, Aziz H, Schoenhoff F, Bedja D, et al. Angiotensin II type 2 receptor signaling attenuates aortic aneurysm in mice through ERK antagonism. Science. 2011;332:361-5.

13. Brooke BS, Habashi JP, Judge DP, Patel N, Loeys B, Dietz HC III. Angiotensin II blockade and aortic-root dilation in Marfan's syndrome. N Engl J Med. 2008; 358:2787-95.

14. Xiong W, Meisinger T, Knispel R, Worth JM, Baxter BT. MMP-2 regulates Erk1/ 2 phosphorylation and aortic dilatation in Marfan syndrome. Circ Res. 2012;110: e92-101.

15. Moberg K, De Nobele S, Devos D, Goetghebeur E, Segers P, Trachet B, et al. The Ghent Marfan Trial_a randomized, double-blind placebo controlled trial with losartan in Marfan patients treated with beta-blockers. Int J Cardiol. 2012;157: $354-8$.

16. Swindle MM, Makin A, Herron AJ, Clubb FJ Jr, Frazier KS. Swine as models in biomedical research and toxicology testing. Vet Pathol. 2012;49:344-56.

17. Endo M, Yamaki S, Hata M, Saiki Y, Tabayashi K. Pulmonary vascular changes induced by unilateral pulmonary venous obstruction. Pediatr Cardiol. 2002;23: 420-5.

18. LaBourene JI, Coles JG, Johnson DJ, Mehra A, Keeley FW, Rabinovitch M. Alterations in elastin and collagen related to the mechanism of progressive pulmonary venous obstruction in a piglet model: a hemodynamic, ultrastructural, and biochemical study. Circ Res. 1990;66:438-56.

19. Chazova I, Robbins I, Loyd J, Newman J, Tapson V, Zhdaov V, et al. Venous and arterial changes in pulmonary veno-occlusive disease, mitral stenosis and fibrosing mediastinitis. Eur Respir J. 2000;15:116-22.

20. Endo M, Yamaki S, Ohmi M, Tabayashi K. Pulmonary vascular changes induced by congenital obstruction of pulmonary venous return. Ann Thorac Surg. 2000; 69:193-7.

21. Sadr IM, Tan PE, Kieran MW, Jenkins KJ. Mechanism of pulmonary vein stenosis in infants with normally connected veins. Am J Cardiol. 2000;86:577-9. A10.

22. Riedlinger WF, Juraszek AL, Jenkins KJ, Nugent AW, Balasubramanian S, Calicchio ML, et al. Pulmonary vein stenosis: expression of receptor tyrosine kinases by lesional cells. Cardiovasc Pathol. 2006;15:91-9.

23. Giannotta M, Trani M, Dejana E. VE-cadherin and endothelial adherens junctions: active guardians of vascular integrity. Dev Cell. 2013;26:441-54.
24. Dejana E, Vestweber D. The role of VE-cadherin in vascular morphogenesis and permeability control. Prog Mol Biol Transl Sci. 2013;116:119-44.

25. Chai Q, Wang XL, Zeldin DC, Lee HC. Role of caveolae in shear stress-mediated endothelium-dependent dilation in coronary arteries. Cardiovasc Res. 2013;100: 151-9.

26. Rudini N, Felici A, Giampietro C, Lampugnani M, Corada M, Swirsding K, et al VE-cadherin is a critical endothelial regulator of TGF-beta signalling. EMBO J 2008;27:993-1004

27. Yang X, Wang F, Lau WB, Zhang S, Zhang S, Liu H, et al. Autoantibodies isolated from preeclamptic patients induce endothelial dysfunction via interaction with the angiotensin II AT1 receptor. Cardiovasc Toxicol. 2014;14:21-9.

28. Holm TM, Habashi JP, Doyle JJ, Bedja D, Chen Y, van Erp C, et al. Noncanonical TGFbeta signaling contributes to aortic aneurysm progression in Marfan syndrome mice. Science. 2011;332:358-61.

29. Yang HH, Kim JM, Chum E, van Breemen C, Chung AW. Long-term effects of losartan on structure and function of the thoracic aorta in a mouse model of Marfan syndrome. Br J Pharmacol. 2009;158:1503-12.

30. Habashi JP, Judge DP, Holm TM, Cohn RD, Loeys BL, Cooper TK, et al. Losartan, an AT1 antagonist, prevents aortic aneurysm in a mouse model of Marfan syndrome. Science. 2006;312:117-21.

31. Friedberg MK, Cho MY, Li J, Assad RS, Sun M, Rohailla S, et al. Adverse biventricular remodeling in isolated right ventricular hypertension is mediated by increased transforming growth factor-beta 1 signaling and is abrogated by angiotensin receptor blockade. Am J Respir Cell Mol Biol. 2013;49:1019-28.

\section{Discussion}

Dr Duke E. Cameron (Baltimore, Md). I have no disclosures. Dr Zhu, I congratulate you and your coauthors on this provocative and potentially groundbreaking study on the mechanism and treatment of PVS. Your group has already made substantial contributions to the characterization of this lesion, you have created a powerful animal model, and now you have presented to us a novel nonsurgical treatment.

Your present study suggests that TGF- $\beta$ signaling might be important to the venous intimal hyperplasia that characterizes this lesion and that a strategy of angiotensin II blockade might thwart the progressive myeloproliferation that occurs upstream to the obstruction, similar to how the blockade can slow and perhaps even prevent aneurysmal changes in the ascending aorta in patients with Marfan syndrome.

I have 3 questions.

You pointed out in your report, although it was not presented here, that when you measured the TGF- $\beta$ levels in the banded and losartan-treated piglets, they actually were not elevated in the setting of banding, although you did see that in other models of pulmonary artery banding that you had studied before. I am curious to know whether you think this was a problem of perhaps measuring TGF- $\beta$ at the wrong location or the wrong time, or have you actually revealed a new mechanism of losartan that could account for that finding? So, in other words, what are your thoughts about how losartan is actually working?

My second question then is that losartan was given before the pulmonary venous changes occurred, and I am interested to know whether you are planning to use this model in a more chronic phase to determine whether losartan truly prevents the changes or has just delayed them and they did not appear within the first 8 weeks.

Of course, we would also like to know whether, if losartan is given once the changes have already occurred, could it actually reverse some of those changes, such as it appears to do in some aneurysmal disease? 
Finally, my last question, which I think everyone in the audience wants to know, is, have you given losartan to any of your clinical patients? If so, to what group of patients and do you have any findings to share with us?

Again, I congratulate you on a spectacular study.

Dr Zhu. Thank you very much, Dr Cameron, thanks for your comments.

For your first question about the TGF- $\beta$ discrepancy between our present study and the previous study, we did show that TGF$\beta$ was upregulated in our previous model. However, we did not find that change in the present study. I think there are several reasons. First, we used a slightly different model. In our previous animal model, we banded all the pulmonary veins. However, in the present study, we left the right middle pulmonary vein unbanded. We intended to leave the right middle pulmonary vein unbanded to improve survival and also to provide an intra-animal control. Thus, the present model was less severe than the previous one. Actually from the hemodynamic measurements, we found the mean pulmonary arterial pressure in the present model to be slightly lower than that in the previous model.

The second reason might have been related to specimen collection. In the present model, we collected the pulmonary vein samples much more distally than in the previous model, because we wanted to focus on the upstream pulmonary vein.

Finally, I think, actually, for TGF- $\beta$, for its role in PVS development, we are still not quite sure. More mechanism studies are required, and our research group is investigating the underlying mechanism now. TGF- $\beta$ could be involved in the early phase; for instance, perhaps it initiates the process of pulmonary vein stenosis, but perhaps it is not required to maintain the PVS. Thus, we might have missed the timing to detect when the TGF- $\beta$ is elevated. Therefore, I think will be good for us to evaluate the TGF- $\beta$ levels at different points in a chronic model.

That brings us to your second question. In the present study, we gave losartan before the development of PVS.

We did not give losartan treatment for established PVS. From our previous study, we had found that PVS starts at around 3 weeks after the banding procedure. We saw some flow turbulence of the banded pulmonary veins on echocardiography. Also, when we killed the pigs at 3 weeks after the operation, we found some pathologic changes. Thus, I think in future studies, we will start giving losartan 3 weeks after banding to test the ability of losartan to reverse established disease. That is our next step. I think it will be very interesting to proceed with this experiment.
The last question I think is the one all of us are mostly interested in-whether we can bring losartan into clinical use. Currently, we have not used losartan clinically; however, we are interested in doing so because losartan is a widely used Food and Drug Administration-approved medication with a good safety profile, and the dosage we used in the present study was within the normal clinical use.

We have not started the clinical trial for a few reasons. First, in the present study, we used losartan before the PVS was established, which is not very similar to the most common clinical setting. The translation of this model to a clinical study would likely entail prophylactic use of losartan in every patient with total anomalous pulmonary venous drainage at risk of PVS. However, we know that the incidence of PVS after total anomalous pulmonary venous drainage repair is only around $10 \%$. So, if we wanted to detect whether losartan has a beneficial prophylactic effect, we might need a few hundred patients to have enough power to identify the effects. In a single center, we would not have enough patients. We would need a multiple center clinical trial to obtain enough patients.

We could consider using losartan only in high-risk patients to increase the likelihood of detecting a beneficial effect; however, these patients are also still very few, and sometimes it is a bit difficult to predict which patients will develop PVS and which will not.

We are very interested in the use of losartan to treat established PVS. I think before we can start the clinical trial of those patients, we will still need some more supportive evidence from animal studies, and this is also our next step.

Finally, for the potential clinical trial of this rare, but relentless, pulmonary vein disease, we need multiple centers to enroll enough patients, and all of you are welcome to join this study.

Dr John E. Mayer, Jr (Boston, Mass). It seems to me, unless I missed something in your presentation, you have sort of a control, namely, that right middle pulmonary vein that you did not band. How were your findings different in that area compared with the other areas in which the veins were banded?

Dr Zhu. Thank you, that is a very good question. Actually, we left the right middle pulmonary vein unbanded. From our findings, when we killed the pigs, morphologically, the right middle pulmonary vein was dilated. It indicates pulmonary flow distribution to the right middle pulmonary vein, which can decompress the heart. We also performed pathologic tests, and we did not find the same intimal hyperplasia in this unbanded pulmonary vein as was seen in the banded veins. Possibly, a longer study duration might have allowed us to detect histologic changes in the unbanded pulmonary veins. 